\section{Protocolo de atención odontológica en pacientes pediatricos con sindrome de down}

Protocol of dental care in pediatric patients with down syndrome

Suleyka Briggitte Paladines Pardo

Egresada de la Universidad Católica de

Santiago de Guayaquil,

suleykapaladines@hotmail.com

https://orcid.org/0000-0002-0408-0891

Adriana Rocio Amado Schneider

Especialista en Odontopediatría y

Ortodoncia, Docente Universidad Católica

de Santiago de Guayaquil.

adrianaamados@hotmail.com

https://orcid.org/0000-0002-3478-7505

Guayaquil - Ecuador

http://www.jah-journal.com/index.php/jah

Journal of American health

Octubre - Diciembre vol. 3. Num. 3-2020

Esta obra está bajo una Licencia Creative Commons

Atribución-NoComercial-CompartirIgual 4.0 Internacional.

RECIBIDO: 8 DE AGOSTO 202

ACEPTADO: 18 DE SEPTIEMBRE 2020

PUBLICADO: 4 DE OCTUBRE 2020

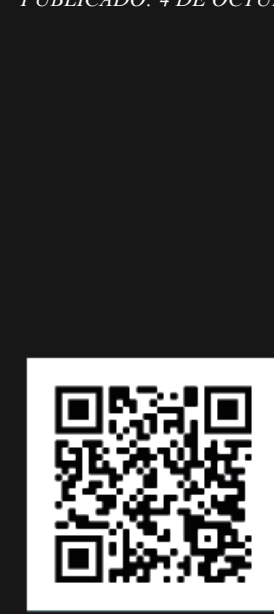

\section{RESUMEN}

Objetivo: Determinar cuál es el protocolo de atención odontológica en pacientes pediátricos con síndrome de Down. Materiales y métodos: Es una investigación de tipo descriptivo no experimental transversal retrospectivo con enfoque cualitativo, se utilizaron meta buscadores como Pubmed - Medline, además Scopus como buscador bibliométrico y la plataforma virtual de la UCSG. Resultados: Los pacientes con síndrome de Down presentaron 56\% hipotonía muscular, $88 \%$ macroglosia, en un 56.23\%, enfermedad periodontal, $88 \%$ de los niños presentaron el hábito de la respiración bucal, en un $53.5 \%$ de los niños se cepillaban dos veces al día, mientras que el $62.33 \%$ de los niños necesitaron supervisión de los padres para poder realizar los hábitos de higiene dental. El $63.2 \%$ de los niños había visitado un odontólogo al menos una vez en su vida, por lo cual el tratamiento más realizado en una consulta dental en un $85,49 \%$ es la profilaxis dental. Conclusión: Los pacientes con síndrome de Down a menudo tienen muchas dificultades para encontrar odontólogos que puedan brindar un tratamiento multidisciplinario, por lo cual se sugiere que se realicen más estudios sobre las alternativas de tratamiento en donde incluya a padres y familiares a participar de la higiene bucal de los niños desde su nacimiento, promoviendo así la participación y la prevención por parte de los padres.

PALABRAS CLAVE: síndrome de Down, discapacidad, atención odontológica, enfermedades bucales.

\section{ABSTRACT}

Objective: To determine which the care dental protocol is for Down syndrome 
pediatric patients. Materials and methods: It is a descriptive, non-experimental, crosssectional, retrospective research with a qualitative approach, using metasearch engines such as Pubmed - Medline, also Scopus as a bibliometric search engine and the virtual platform from the UCSG. Results: Down syndrome patients presented $56 \%$ of muscular hypotonia, $88 \%$ showed macroglossia and $56.23 \%$ showed periodontal disease, $88 \%$ of the children presented the habit of mouth breathing, $53.5 \%$ of children brushed their teeth twice a day while $62.33 \%$ of the children required parental supervision in order to achieve good dental hygiene. $62.3 \%$ of children had visited an odontologist at least once in their lives which is why the most performed treatment in a dental appointment has been dental prophylaxis in about $85.49 \%$ of children. Conclusion: Down syndrome patients often have difficulties in finding odontologists who can provide a multidisciplinary treatment, which is why it is suggested that more studies are done regarding the treatment alternatives that include parents and relatives to participate in dental hygiene since birth, thus promoting the participation and prevention by parents

KEYWORDS: Down syndrome, disability, dental care, oral diseases

\section{INTRODUCCIÓN}

El síndrome de Down es una alteración congénita, que se caracteriza por tener una réplica del cromosoma par 21, el cual también se lo denomina trisomía 21.(1) Es reconocido en el año 1866 por John Langdon Haydon Down de ahí su nombre, este investigador logro describir sobre el trastorno genético, sin embargo, no pudo demostrar que causas lo producían. En la actualidad está demostrado que este síndrome puede deberse a tres causas, como son la falta de disyunción en la meiosis, falta de disyunción en la mitosis o mediante una translocación desequilibrada.(2).(3) Sin embargo también puede deberse a una serie de factores que incluyen la edad de la mamá a partir de los 35 años en adelante.(4) Algunos estudios relacionados a personas con síndrome de Down (SD) llegan a una conclusión que nace uno por cada 700 nacidos vivos, mientras que la cifra en el Ecuador es uno por cada 550 nacidos vivos. $(5,6)$ Estos pacientes por lo general presentan irregularidades en cuanto a su desarrollo físico, como las estructuras craneofaciales, afectando a labios, mucosa, dentición, además de discapacidad intelectual y de crecimiento, lo cual lo hace más vulnerable en su desenvolvimiento con la sociedad.(1)(7)(8,9) También pueden presentar mayor susceptibilidad a desarrollar enfermedad periodontal y caries dental como consecuencia de hábitos de higiene no supervisados. $(1,7,8)$

La historia clínica del paciente debe estar correctamente llena, es decir deberá contener todos los datos del paciente, especialmente las enfermedades sistémicas que padece(12) Por otro lado, las consultas dentales deberán ser lo más cortas posibles, para evitar todo tipo de fatiga y estrés que pueda ocasionar al niño con síndrome de $\operatorname{Down}(7,12)$. La familia de los niños con síndrome de Down cumplen una función muy importante, la cual va a tener una gran respuesta 
al tratamiento dental del niño, de ellos depende el desarrollo de las habilidades y su higiene dental. $(2,13)$

En la actualidad el profesional dental cumple una función multidisciplinaria en la rehabilitación de los pacientes con síndrome de Down, debido a sus conocimientos generales de todas las enfermedades que estos pacientes puedan manifestar, además deben ofrecerle un tratamiento digno que proporcione un bienestar y una integración de los niños con el entorno social. $(6)(14,15)$ Es necesario que el odontólogo enseñe a conservar los hábitos adecuados de higiene para mantener salud oral en los niños, además eliminar la causa que provoca los hábitos orales, motivando también a los padres del niño informándoles sobre la importancia que tiene la prevención de enfermedades que afectan a la cavidad oral y promoviendo una alimentación sana. $(16,17)$ El objetivo de esta investigación a través de la revisión literaria de artículos científicos es poder determinar cuál es el protocolo de atención odontológica en pacientes pediátricos con síndrome de Down.

\section{MATERIALES Y MÉTODOS}

Criterios para la selección de estudios.

Esta investigación es de tipo descriptivo- no experimental transversal retrospectivo que tiene un enfoque cualitativo, en la cual se analizó mediante la revisión de literatura de 150 artículos científicos, los cuales a través de los criterios de inclusión y exclusión se seleccionaron 47 artículos, la realidad de la situación que enfrentan los niños con Síndrome de Down en la consulta dental, se utilizaron meta buscadores como Pubmed - Medline, además se utilizó Scopus como buscador bibliométrico que proporciono el índice de impacto y calidad de los autores según los cuartiles , y como herramienta la plataforma virtual de la UCSG.

Entre los criterios de inclusión y exclusión se consideraron artículos que mencionen a niños con Síndrome de Down en edades entre 0-12 años que analicen hábitos de higiene oral, la atención y tratamiento odontológico.

Se utilizó palabras claves como: síndrome de Down, discapacidad, atención odontológica, enfermedades bucales.

Se incluyeron artículos en idioma español, inglés, portugués y francés.

En este artículo se analizaron las siguientes variables: Características del paciente síndrome de Down, Enfermedades más comunes en la cavidad bucal en niños con síndrome de Down, Hábitos Orales más frecuentes en niños con síndrome de Down, Frecuencia del cepillado en los niños con síndrome de Down, Supervisión de padres y cuidadores en la higiene de los niños con síndrome de Down, Tratamientos odontológicos más frecuentes en los niños con síndrome de Down, Visitas odontológicas en los niños con síndrome de Down.

\section{RESULTADOS}

En la búsqueda de información, se encontró 150 artículos científicos, en los cuales se excluyeron 53 por presentar información con poca relevancia científica, 25 por presentar problemas de acceso y 25 por irregularidades en cuanto el tema y el contenido, mediante los criterios de inclusión y exclusión se seleccionaron 47 artículos que sirvieron para la realización de este proyecto, además los artículos seleccionados representan al 70\% de los últimos 10 años 


\begin{tabular}{|c|c|c|c|c|c|c|c|}
\hline REFERENCIA & AÑO & Hipotonía & Hidrocefalia & $\begin{array}{l}\text { Retraso del desarrollo } \\
\text { psicomotor y } \\
\text { Discapacidad } \\
\text { intelectual }\end{array}$ & $\begin{array}{l}\text { Cardiopatías- } \\
\text { Problemas } \\
\text { inmunológicos }\end{array}$ & $\begin{array}{l}\text { Problemas } \\
\text { hematológicos }\end{array}$ & $\begin{array}{l}\text { Problemas } \\
\text { ortopédicos }\end{array}$ \\
\hline (18) & 1992 & $50 \%$ & $\begin{array}{l}-50 \% \text { de los } \\
\text { casos }\end{array}$ & $\begin{array}{l}50 \% \text { de discapacidad } \\
\text { intelectual y psicomotor }\end{array}$ & $x$ & $X$ & $X$ \\
\hline (19) & 1997 & $X$ & $X$ & $\begin{array}{l}30 \% \text { de discapacidad } \\
\text { intelectual }\end{array}$ & $\begin{array}{l}40 \% \text { cardiopatías } \\
\text { congénitas }\end{array}$ & $\begin{array}{l}\text { Riesgo de } \\
\text { Leucemia 0,5\% }\end{array}$ & $X$ \\
\hline (5) & 2007 & $X$ & $X$ & $\begin{array}{l}51 \% \text { de discapacidad } \\
\text { intelectual } \\
38 \% \text { desarrollo } \\
\text { psicomotor }\end{array}$ & $\begin{array}{l}84 \% \text { cardiopatías } \\
\text { congénitas } \\
\text { P.IMN. } 54 \% \\
\text { hipotiroidismo }\end{array}$ & $x$ & $X$ \\
\hline (20) & 2008 & $x$ & $x$ & $\begin{array}{l}50 \% \text { de discapacidad } \\
\text { intelectual }\end{array}$ & $\begin{array}{l}40-60 \% \text { cardiopatías } \\
\text { congénitas } \\
\text { P.IMN } 17 \% \\
\text { hipotiroidismo }\end{array}$ & $\begin{array}{l}\text { Riesgo de } \\
\text { Leucemia } \\
1-1,5 \%\end{array}$ & $\begin{array}{l}10-30 \% \\
\text { (10\%sintomático) }\end{array}$ \\
\hline (7) & 2011 & $100 \%$ & $X$ & $\begin{array}{l}50 \% \text { de discapacidad } \\
\text { intelectual }\end{array}$ & $\begin{array}{l}40-60 \% \text { cardiopatía } \\
\text { congénita }\end{array}$ & $\begin{array}{l}\text { Riesgo de } \\
\text { Leucemia 12-40\% }\end{array}$ & $X$ \\
\hline (4) & 2014 & $x$ & $x$ & $X$ & $\begin{array}{l}35-50 \% \text { cardiopatía } \\
\text { congénita } \\
\text { Hipotiroidismo } 30 \%\end{array}$ & $\begin{array}{l}\text { Riesgo de } \\
\text { Leucemia 30\% }\end{array}$ & $x$ \\
\hline (21) & 2017 & $20 \%$ & $x$ & $X$ & $\begin{array}{l}40 \% \text { cardiopatía } \\
\text { congénita }\end{array}$ & $x$ & $x$ \\
\hline
\end{tabular}

Tabla 1.

En la tabla 1 como se puede observar, se muestran los resultados de las características generales más prevalentes en los niños con síndrome de Down: un 57\% con hipotonía muscular, un $51.08 \%$ tienen el riesgo de padecer cardiopatías congénitas, 50\% hidrocefalia, en un $46 \%$ de discapacidad intelectual, $33.6 \%$ problemas inmunológicos, en un $14.5 \%$ de los casos pueden presentar leucemia, y en un $20 \%$ suelen estar asociados a tener problemas ortopédicos. $(4,5,7,18-21)$

\begin{tabular}{|c|c|c|c|c|c|c|c|}
\hline $\begin{array}{l}\text { REFER } \\
\text { ENCIA }\end{array}$ & AÑO & Macroglosia & $\begin{array}{l}\text { Lengua } \\
\text { fisurada }\end{array}$ & $\begin{array}{l}\text { Amígdalas } \\
\text { Hipertrófica } \\
\text { s }\end{array}$ & $\begin{array}{l}\text { Alteración en el } \\
\text { número de dientes }\end{array}$ & $\begin{array}{l}\text { Alteración en el } \\
\text { tamaño de los } \\
\text { dientes }\end{array}$ & $\begin{array}{l}\text { Molares } \\
\text { alteraciones } \\
\text { estructurales }\end{array}$ \\
\hline (18) & 1992 & $\begin{array}{l}88 \% \text { de los } \\
\text { casos }\end{array}$ & $\begin{array}{l}55 \% \text { de } \\
\text { los casos }\end{array}$ & $\begin{array}{l}65-70 \% \text { de } \\
\text { los casos }\end{array}$ & $\begin{array}{l}\text { Agenesia o } \\
\text { Anodoncia 23- } \\
47 \% \text { de los } \\
\text { casos } \\
\text { Hipodoncia 12- } \\
17 \% \text { de los } \\
\text { casos }\end{array}$ & $\begin{array}{l}\text { Microdoncia } \\
35-55 \% \text { de } \\
\text { los casos }\end{array}$ & $\begin{array}{l}\text { Hipotaurodonticos } \\
14 \% \text { de los casos } \\
\text { - } \quad \text { Meso } 4 \% \text { de los } \\
\text { casos } \\
\text { - Hipertaurodontico } 1 \\
\% \text { de los casos }\end{array}$ \\
\hline (19) & 1997 & $X$ & $X$ & $X$ & $\begin{array}{l}\text { - } \quad \text { Anodoncia } \\
50 \% \\
\text { - } \quad \text { Supernumerarios } \\
+0.3 \%\end{array}$ & $\begin{array}{l}\text { Microdoncia } \\
35-55 \% \text { de } \\
\text { los casos }\end{array}$ & $\begin{array}{l}\text { - Presencia en general } \\
\text { de Taurandontismo } \\
0.54 \text { a } 5.6 \%\end{array}$ \\
\hline
\end{tabular}


(22)

$2007 \quad x \quad X \quad X$

- Anodoncia

- Microdoncia

- Presencia en general

$34,69 \%$

2,04

de Taurodontismo

$85.71 \%$

(4) $2014 \quad \mathrm{X}$

$X$

- $\quad$ Agenesia 53\%

- Microdoncia

- Presencia en general

$35-55 \%$ de Taurodontismo

$0.54-5.6 \%$

(23)

$2019 \quad X$

X

$\mathrm{X}$

- Agenesia $65 \% \quad X$

- Oligodoncia

$65 \%$

\section{TABLA 2: Características de la cavidad oral del paciente síndrome de Down}

En la tabla 2 se muestran los resultados de las características de la cavidad bucal más prevalentes en el niño con síndrome de Down en un porcentaje de $88 \%$ de los niños presentan macroglosia, en un $67.5 \%$ presentan lengua fisurada, estos niños suelen presentar alteraciones del número de dientes en un $47.53 \%$ anodoncia, en un $14.5 \%$ hipodoncia, $65 \%$ oligodoncia y $0.3 \%$ dientes supernumerarios. Además, suelen presentar problemas con la alteración del tamaño de los dientes en un $34.26 \%$ Microdoncia, y un $30.61 \%$, de manera general en problemas en los molares con alteraciones estructurales como son los dientes con taurodontismos, un $14 \%$ hipotaurodontismo, $4 \%$ mesotaurodontismo y $1 \%$ hipertaurodontismo. . $(3,10,11,14,15)$

GRÁFICO 3: Resultado de las enfermedades más comunes en la cavidad bucal en niños con síndrome Down 
En el gráfico 3 podemos observar que las enfermedades más comunes que prevalecen en la cavidad bucal son la enfermedad periodontal con un $56,23 \%$ y la caries dental en un $35.37 \%$. $(2,10,16-25)$

GRÁFICO 4: Hábitos orales más frecuentes en niños con síndrome de Down

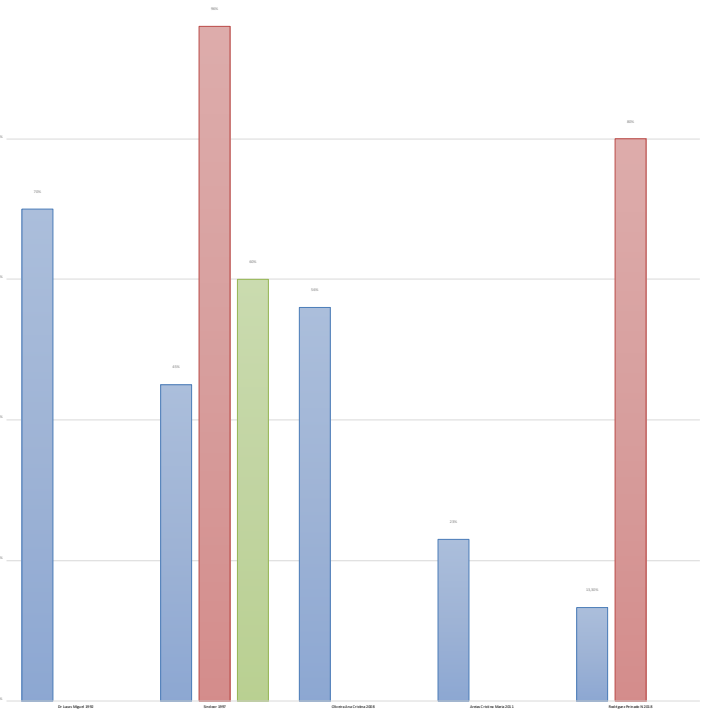

En el gráfico 4 se observan que los hábitos más comunes dentro la cavidad bucal del niño con síndrome de Down en un $88 \%$ se encuentra la respiración bucal, seguido por un $60 \%$ de masticación inadecuada, y un 41,46\% de bruxismo.(10, 11, 19, 21,26)

GRÁFICO 5: Frecuencia de cepillado y supervisión de padres y cuidadores en la higiene. 


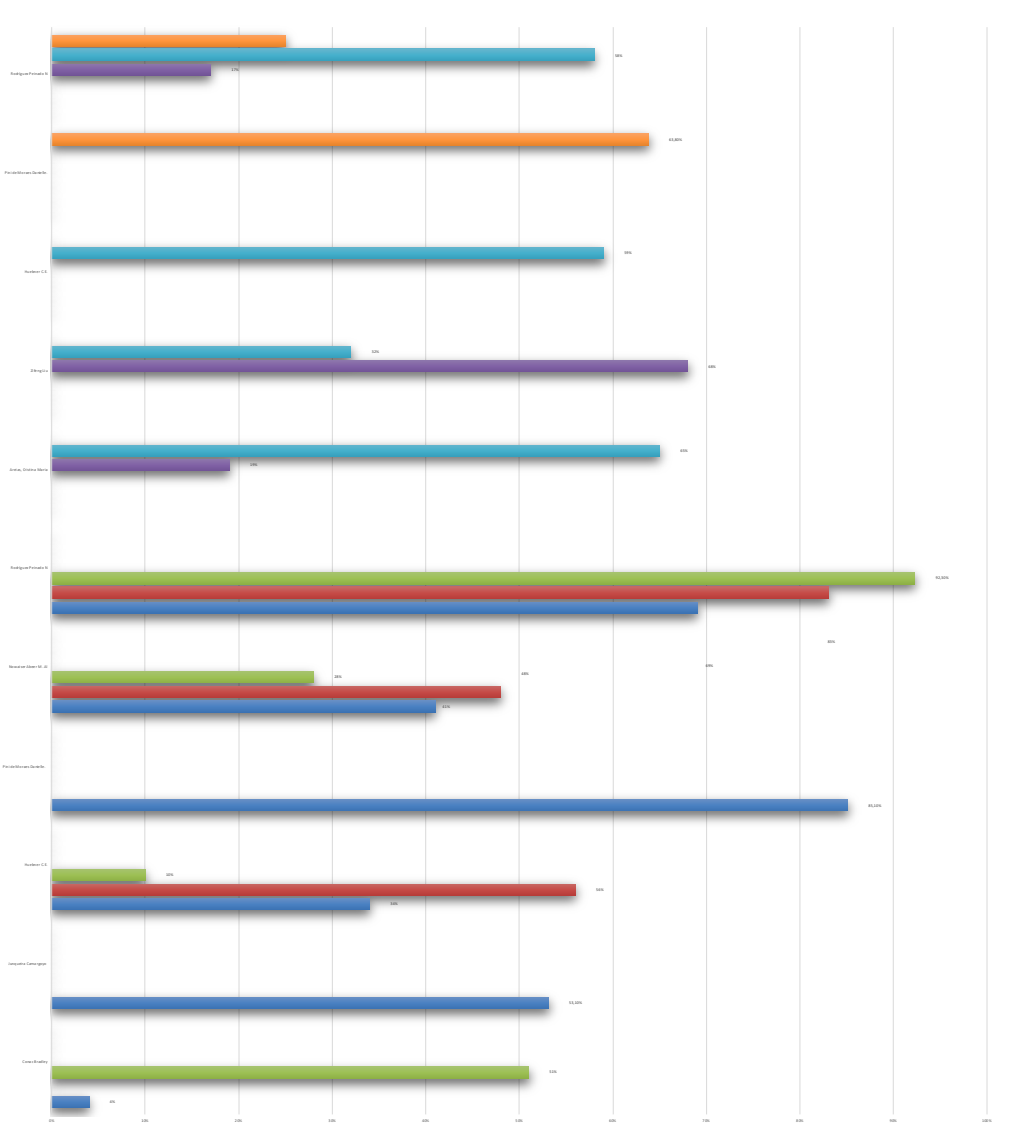

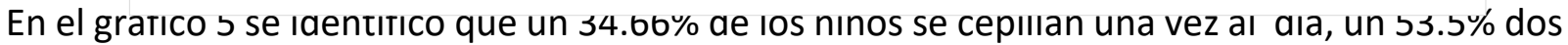
veces al día, y un $30.60 \%$ más de dos veces al día, además se identificó que un $62.33 \%$ de los niños con síndrome de Down necesitaron supervisión de los

padres o cuidadores para realizar el cepillado dental, mientras que un $45.32 \%$ de los niños no necesitaron ninguna ayuda, sin embargo, un $47.7 \%$ necesitaron de una higiene asistida.( 9 , $16,21-23,26-28)$

GRÁFICO 6: Tratamientos odontológicos más frecuentes en los niños con síndrome de Down 


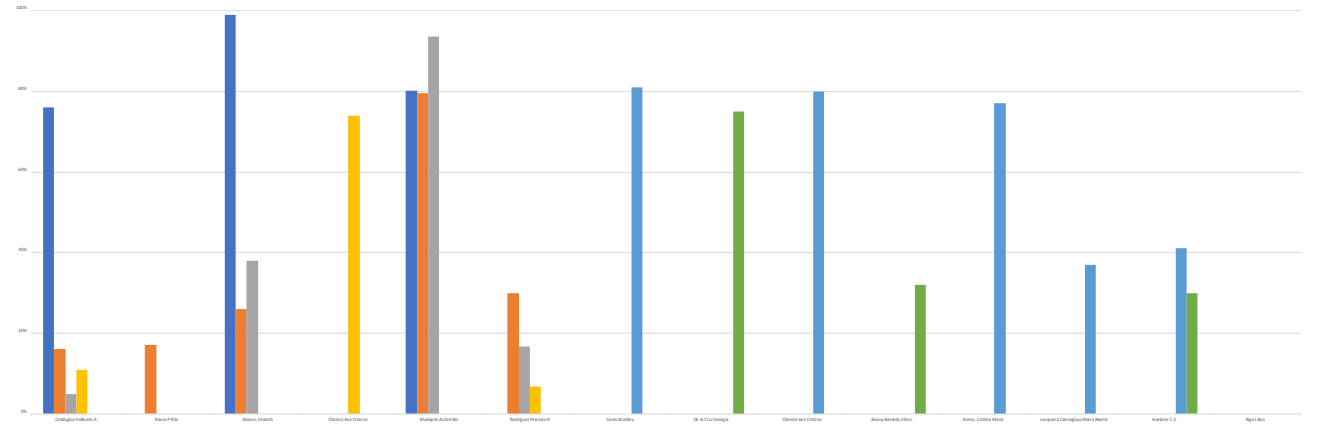

\section{DISCUSIÓN}

El comportamiento de los síntomas clínicos durante el climaterio de las pacientes estudiadas, coincide con lo descrito por la mayoría de los autores, que plantean que los síntomas vasomotores son los más frecuentes y pueden observarse sofocos y oleadas de calor entre un 80\% en el estudio realizado en el 2016 en cuba fue del $75 \%$ y un $85 \%$ pero aquí no fueron utilizados los niveles de intensidad (15). Con respecto al estado de ánimo de las pacientes un alto porcentaje refiere estar dentro de lo normal es decir sin alteraciones.Además, las pacientes con antecedentes de depresión, en particular durante episodios relacionados con la reproducción, también parecen tener mayor riesgo de depresión durante el período en

estudio (16) .En lo referente a la sexualidad, un 43\%\% Manifestó ausencia de problemas sexuales y otro 29\% problemas leves (17). En un estudio realizado en cuba en el año 2017 .La ansiedad y la depresión fueron las manifestaciones psíquicas más frecuentes; y en la esfera sexual predominó la disminución del deseo sexual en las peri menopáusicas $(25.6 \%)$ y la insatisfacción en las postmenopáusicas (58.3\%). En el período posmenopáusico se intensifican los cambios genitourinarios, sobre todo la sequedad vaginal, la caída del vello axilar y púbico y la incontinencia urinaria, que a su vez pueden incrementar las disfunciones sexuales. En nuestro estudio las pacientes manifestaron problemas urinarios de leves a moderado en un $43 \%$ cabe recalcar que todos los grupos presentaron problemas reflejados en las siguientes 
escalas. Así también como ausencia. Existen receptores de estrógenos y progestágenos en la vagina, en la vejiga, en la uretra y en la musculatura del suelo pélvico por lo que, el descenso de los niveles de estrógenos en sangre incide en dichas estructuras con receptores para estas hormonas.

Aparecen las alteraciones menstruales y se manifiestan de forma más evidente los síntomas característicos del síndrome climatérico: alteraciones neurovegetativas con síntomas vasomotores de sofocos, cambios de humor, sudación e insomnio, pueden presentarse síntomas a nivel genitourinario, como atrofia y sequedad vaginal, cambios en su sexualidad, así como pueden aparecer algunas patologías que se deben prevenir, las más frecuentes son la osteoporosis y la enfermedad cardiovascular (20)

Aquellas que experimentan otros síntomas, como sofocos e insomnio, parecen encontrarse especialmente en riesgo de presentar depresión durante este período. Además, las pacientes con antecedentes de depresión, en particular durante episodios relacionados con la reproducción, también parecen tener mayor riesgo de depresión durante el período en estudio (21).

Con respecto al IMC un alto porcentaje de las mujeres en la etapa del climaterio presenta sobre peso en el $43 \%$ La culpable del incremento de peso tras el climaterio podría ser la hormona folículo estimulante, que al aumentar sus niveles tras la menopausia, provoca menor gasto calórico y mayor adiposidad (22).

\section{CONCLUSIONES}

En conclusión, los niños con síndrome de Down tienen muchas dificultades en recibir atención odontológica o encontrar odontólogos que se encarguen de proporcionar un tratamiento multidisciplinario, por lo cual se sugiere o recomienda que se realicen más estudios en donde los niños con SD reciban más participación e inclusión y más alternativas de tratamiento, involucrando a padres o familiares a participar de la higiene bucal de sus niños desde el nacimiento, promoviendo la prevención de futuras enfermedades.

\section{REFERENCIAS}

1. Maritza Peña Sisto KRG, Rafael Alberto Clavería Clark GRVP, Reina de la Caridad García Díaz. salud bucal en paciente con sindrome de down segun actitud de sus tutores legales. MEDISAN [Internet]. $21 . \quad$ Disponible en: http://scielo.sld.cu/scielo.php?script=sci_arttext\&pid=S1029-30192017000700010

2. Dra. Katiuska Rodríguez Guerrero MsCRACC, MsC. Maritza Peña Sisto. Algunas características clinicoepidemiológicas del síndrome de Down y su repercusión en la cavidad bucal. MEDISAN [Internet]. $19 . \quad$ Disponible en: http://scielo.sld.cu/scielo.php?script=sci_arttext\&pid=S1029-30192015001000013

3. Lubec G, Engidawork E. The brain in Down syndrome (TRISOMY 21). J Neurol. octubre de 2002;249(10):1347-56. 
4. Clemente DVG. Salud oral en el niño con Síndrome de Down: Protocolo de intervención [Internet]. Gaceta Dental. 2014 [citado 3 de junio de 2020]. Disponible en: https://gacetadental.com/2014/02/salud-oral-en-el-nino-con-sindrome-de-down-protocolode-intervencion-47263/

5. Andrade González X. Caracterización clínica y citogenética del retraso mental y del desarrollo psicomotor en pacientes atendidos en el servicio de consulta externa de genética médica del hospital Roberto Gilbert Elizalde, Guayaquil - Ecuador. Medicina (Guayaquil). 2007;7-17.

6. Barros ALO, Barros AO, Barros GL de M, Santos MTBR. Burden of caregivers of children and adolescents with Down Syndrome. Cien Saude Colet. noviembre de 2017;22(11):3625-34.

7. Abanto J, Ciamponi AL, Francischini E, Murakami C. Medical problems and oral care of patients with Down syndrome: a literature review. :7.

8. Diéguez-Pérez M, de Nova-García M-J, Mourelle-Martínez MR, Bartolomé-Villar B. Oral health in children with physical (Cerebral Palsy) and intellectual (Down Syndrome) disabilities: Systematic review I. J Clin Exp Dent. julio de 2016;8(3):e337-343.

9. Saccomanno S, Martini C, D'Alatri L, Farina S, Grippaudo C. A specific protocol of myofunctional therapy in children with Down syndrome. A pilot study. Eur J Paediatr Dent. septiembre de 2018;19(3):243-6.

10. Ghanim A, Elfrink M, Weerheijm K, Mariño R, Manton D. A practical method for use in epidemiological studies on enamel hypomineralisation. Eur Arch Paediatr Dent. junio de 2015;16(3):235-46.

11. Stefanini M, Sangiorgi M, Roncati M, D’Alessandro G, Piana G. Effect on plaque control in children patients with Down syndrome using Digital Brush with or without chlorhexidine: a randomized clinical trial. Spec Care Dentist. abril de 2016;36(2):66-70.

12. Mubayrik AB. The Dental Needs and Treatment of Patients with Down Syndrome. :14.

13. Perdomo BJ, Torres D, Paredes Y. Síndrome de Down e higiene bucal: Lineamientos para padres, cuidadores y docentes. Revista Venezolana de Investigación Odontológica. 2014;2(2):156-69.

14. Manejo odontológico en paciente con Síndrome de Down [Internet]. [citado 18 de mayo de 2020]. Disponible en: https://www.ortodoncia.ws/publicaciones/2019/art-25/\#

15. Monteserín-Matesanz M, Esparza-Gómez GC, García-Chías B, Gasco-García C, CereroLapiedra R. Descriptive study of the patients treated at the clinic «integrated dentistry for 
patients with special needs» at Complutense University of Madrid (2003-2012). Med Oral Patol Oral Cir Bucal. 1 de marzo de 2015;20(2):e211-217.

16. Pini D de M. Oral health evaluation in special needs individuals. :7.

17. Ziegler J, Spivack E. Nutritional and dental issues in patients with intellectual and developmental disabilities. J Am Dent Assoc. abril de 2018;149(4):317-21.

18. Thm L. Analisis estomatologico en pacientes con sindrome de Down. :180.

19. Desai SS. Down syndrome: a review of the literature. Oral Surg Oral Med Oral Pathol Oral Radiol Endod. septiembre de 1997;84(3):279-85.

20. Baum RA, Nash PL, Foster JEA, Spader M, Ratliff-Schaub K, Coury DL. Primary care of children and adolescents with down syndrome: an update. Curr Probl Pediatr Adolesc Health Care. septiembre de 2008;38(8):241-61.

21. Nirmala S. Dental Concerns of Children with Down's Syndrome - An Overview. JPNC [Internet]. 7 de marzo de 2017 [citado 26 de mayo de 2020];6(3). Disponible en: https://medcraveonline.com/JPNC/dental-concerns-of-children-with-downrsquos-syndrome--an-overview.html

22. Moraes MEL de, Moraes LC de, Dotto GN, Dotto PP, Santos LR de A dos. Dental anomalies in patients with down syndrome. Brazilian Dental Journal. 2007;18(4):346-50.

23. Symmetry of Dental Agenesis in Down Syndrome Children - PubMed [Internet]. [citado 10 de junio de 2020]. Disponible en: https://pubmed.ncbi.nlm.nih.gov/30988881/?from_term=dental+treatments+for+children+w ith+syndrome+down\&from_filter=simsearch2.ffrft\&from_pos $=5$

24. Bradley C, McAlister T. The oral health of children with Down syndrome in Ireland. Special Care in Dentistry. 2004;24(2):55-60.

25. Oredugba. Oral health condition and treatment needs of a group of Nigerian individuals with Down syndrome [Internet]. Down Syndrome Research and Practice. 2007 [citado 18 de mayo de 2020]. Disponible en: https://library.down-syndrome.org/en-us/researchpractice/12/1/oral-health-condition-treatment-needs-group-nigerian-individuals-downsyndrome/

26. P RN, Mogollón J. Participación de los padres en el cuidado de la salud bucal de los niños y adolescentes con síndrome de Down. Ciencia Odontológica. 2007;4(2):130-40. 
27. Oliveira AC, Czeresnia D, Paiva SM, Campos MR, Ferreira EF. [Utilization of oral health care for Down syndrome patients]. Rev Saude Publica. agosto de 2008;42(4):693-9.

28. Asokan S, Geethapriya PR, Vijayasankari V. ff. Indian J Dent Res. abril de 2019;30(2):28290.

29. Areias CM, Sampaio-Maia B, Guimaraes H, Melo P, Andrade D. Caries in Portuguese children with Down syndrome. Clinics. 2011;66(7):1183-6.

30. Camargo MBJ, Barros AJD, Frazão P, Matijasevich A, Santos IS, Peres MA, et al. Predictors of dental visits for routine check-ups and for the resolution of problems among preschool children. Rev Saude Publica. febrero de 2012;46(1):87-97.

31. Huebner CE, Milgrom P. Evaluation of a parent-designed programme to support tooth brushing of infants and young children. Int J Dent Hyg. febrero de 2015;13(1):65-73.

32. Hennequin M, Faulks D, Veyrune JL, Bourdiol P. Significance of oral health in persons with Down syndrome: a literature review. Dev Med Child Neurol. abril de 1999;41(4):275-83.

33. Descamps I, Fernandez C, Cleynenbreugel DV, Hoecke YV, Marks L. Dental care in children with Down syndrome: A questionnaire for Belgian dentists. Med Oral Patol Oral Cir Bucal. 2019;7.

34. Rodríguez Peinado N, Mourelle Martínez MR, Diéguez Pérez M, De Nova García MJ. A study of the dental treatment needs of special patients: cerebral paralysis and down syndrome. European Journal of Paediatric Dentistry. 2018;(3):233-8.

35. Al-Nowaiser AM, Al Suwyed AS, Al Zoman KH, Robert AA, Al Brahim T, Ciancio SG, et al. Influence of full mouth rehabilitation on oral health-related quality of life among disabled children. Clin Exp Dent Res. octubre de 2017;3(5):171-8.

36. Liu Z, Yu D, Luo W, Yang J, Lu J, Gao S, et al. Impact of Oral Health Behaviors on Dental Caries in Children with Intellectual Disabilities in Guangzhou, China. International Journal of Environmental Research and Public Health. octubre de 2014;11(10):11015-27.

37. Oliveira AC, Paiva SM, Martins MT, Torres CS, Pordeus IA. Prevalence and determinant factors of malocclusion in children with special needs. Eur J Orthod. agosto de 2011;33(4):4138.

38. Bouvy-Berends EC, Reuland-Bosma W. ['Emily goes to the dentist'. Oral care for individuals with Down syndrome]. Nederlands tijdschrift voor tandheelkunde. 2006; 
39. Rigo L, Dalazen J, Garbin RR, Rigo L, Dalazen J, Garbin RR. Impact of dental orientation given to mothers during pregnancy on oral health of their children. Einstein (São Paulo). junio de 2016;14(2):219-25.

40. Allison PJ, Hennequin M, Faulks D. Dental care access among individuals with Down syndrome in France. Spec Care Dentist. febrero de 2000;20(1):28-34.

41. Porovic S, Zukanovic A, Juric H, Dinarevic SM. ORAL HEALTH OF DOWN SYNDROME CHILDREN IN BOSNIA AND HERZEGOVINA. Mater Sociomed. octubre de 2016;28(5):370-2.

42. dela Cruz GG, Rozier RG, Slade G. Dental screening and referral of young children by pediatric primary care providers. Pediatrics. noviembre de 2004;114(5):e642-652.

43. Faker K, Tostes MA, Paula VAC de. Impact of untreated dental caries on oral healthrelated quality of life of children with special health care needs. Braz Oral Res. 18 de marzo de 2019;32:e117.

44. Lewis CW. Dental care and children with special health care needs: a population-based perspective. Acad Pediatr. diciembre de 2009;9(6):420-6.

45. Ferrary T, Sanchez Ratto N, Martinez D, Alvarez M, Bianchi ML, Benitez MB, et al. Psychoprophylaxis for oral conscious sedation for dental care in Down syndrome adults with behavioral disorder. Spec Care Dentist. julio de 2019;39(4):389-98.

46. Is There Anything to Smile About? A Review of Oral Care for Individuals With Intellectual and Developmental Disabilities - PubMed [Internet]. [citado 11 de junio de 2020]. Disponible en: https://pubmed.ncbi.nlm.nih.gov/22548164/

47. Giraldo-Zuluaga MC, Martínez-Delgado CM, Cardona-Gómez N, Gutiérrez-Pineda JL, Giraldo-Moncada KA, Jiménez-Ruíz PM. Manejo de la salud bucal en discapacitados. Artículo de revisión. :14. 